\title{
Correction: miR-9-5p, miR-124-3p, and miR-132-3p regulate BCL2L11 in tuberous sclerosis complex angiomyolipoma
}

\author{
Yi Cai ${ }^{1,2} \cdot$ Wenda Wang ${ }^{1} \cdot \mathrm{Hao} \mathrm{Guo}^{1} \cdot$ Hanzhong $\mathrm{Li}^{1} \cdot \mathrm{Yu}^{\mathrm{Xiao}}{ }^{3} \cdot \mathrm{Yushi}^{\mathrm{Z}} \mathrm{Khang}^{1}$
}

Published online: 18 October 2018

(c) United States \& Canadian Academy of Pathology 2018

Correction to: Laboratory Investigation 98, 856-870 (2018); https://doi.org/10.1038/s41374-018-0051-6; published online 14 March 2018
Following the publication of this article, the authors noticed an error in Fig. 5c. In the miR-132-3p mimics. This does not affect the results and conclusions of the article. The correct version of Fig. 5 can be found below.

Fig. 5 The role of miR-9-5p, miR-124-3p, and miR-132-3p in the regulation of proliferation and apoptosis in Tsc2 ${ }^{-1-}$ cells. a CCK-8 assays were performed $24,48,72$, and $96 \mathrm{~h}$ after the transfection of Tsc $2^{-1-}$ cells with miR-9-5p, miR-124-3p, or miR-132-3p mimics or the scrambled control. b CCK-8 assays were performed $24,48,72$, and $96 \mathrm{~h}$ after the transfection of Tsc $2^{-l-}$ cells with miR-9-5p, miR-124-3p, or miR-132-3p inhibitors or the negative control. $\mathbf{c}, \mathbf{d}$ Tsc $2^{-l-}$ cells were transfected with equal doses of miR-9-5p, miR-124-3p, or miR-132-3p mimics or the scrambled control. The cell apoptosis profiles were analyzed by flow cytometry. The bi-parametric histogram shows cells in early (bottom right quadrant) and late (top right quadrant) apoptotic states. Viable cells are double negative (bottom left quadrant). c Representative image. d Quantitative analysis. e, f Tsc $2^{-I}$ cells were transfected with equal doses of miR-9-5p, miR-124-3p, or miR-132-3p inhibitors or the negative control. The cell apoptosis profiles were analyzed by flow cytometry. The bi-parametric histogram shows cells in early (bottom right quadrant) and late (top right quadrant) apoptotic states. Viable cells are double negative (bottom left quadrant). e Representative image. f Quantitative analysis

Yushi Zhang

zhangyushi2014@126.com

1 Department of Urology, Peking Union Medical College Hospital, Chinese Academy of Medical Sciences and Peking Union Medical College, 100730 Beijing, China

2 Department of Urology, Xiangya Hospital, Central South University, No.87 Xiangya Road, Changsha City 410008 Hunan Province, China

3 Department of Pathology, Peking Union Medical College Hospital, Chinese Academy of Medical Sciences and Peking Union Medical College, 100730 Beijing, China 
A

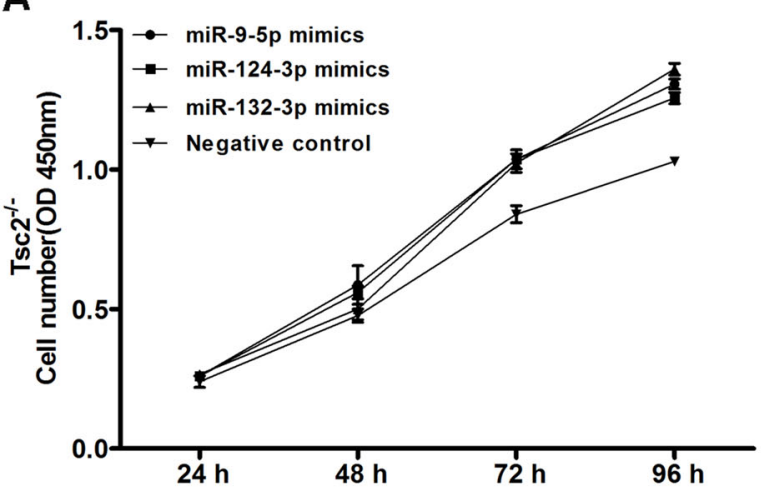

C
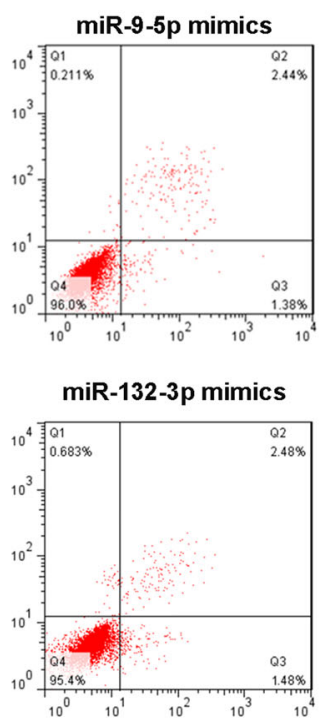

E

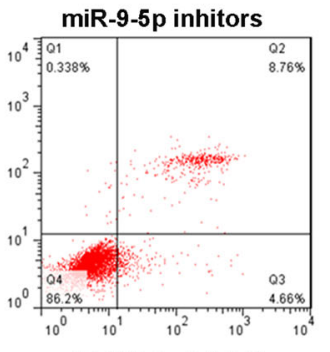

miR-132-3p inhibitors

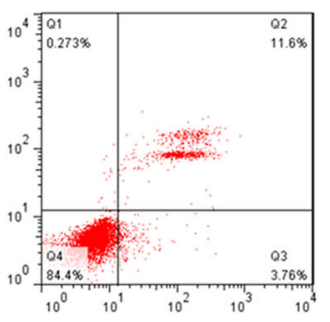

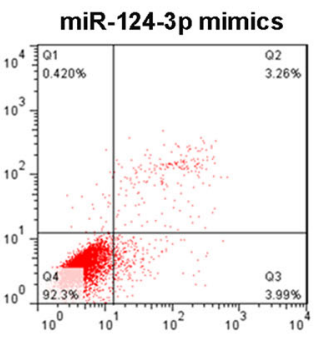

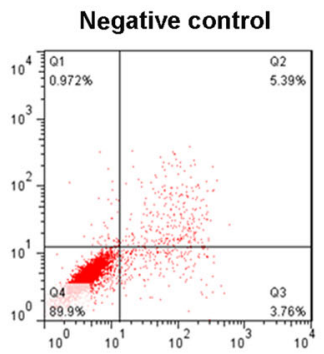

miR-124-3p inhibitors
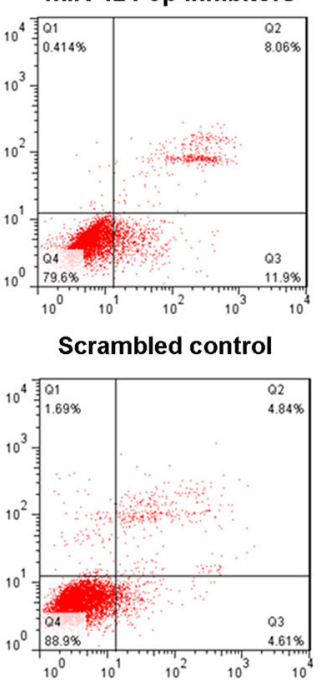

B

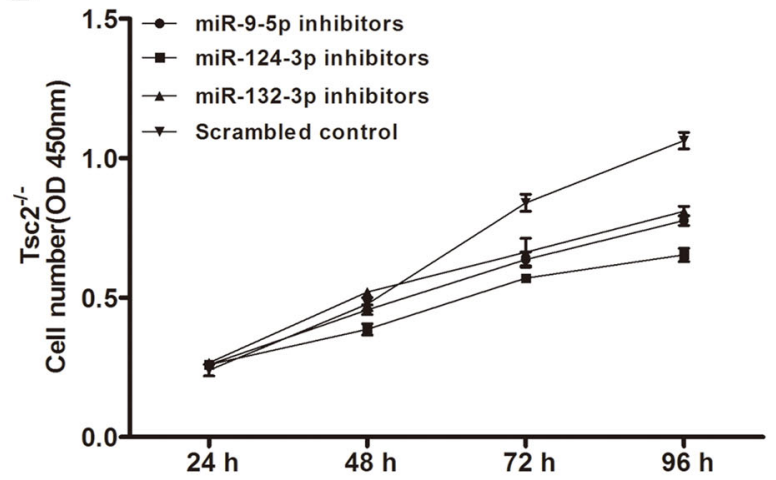

D

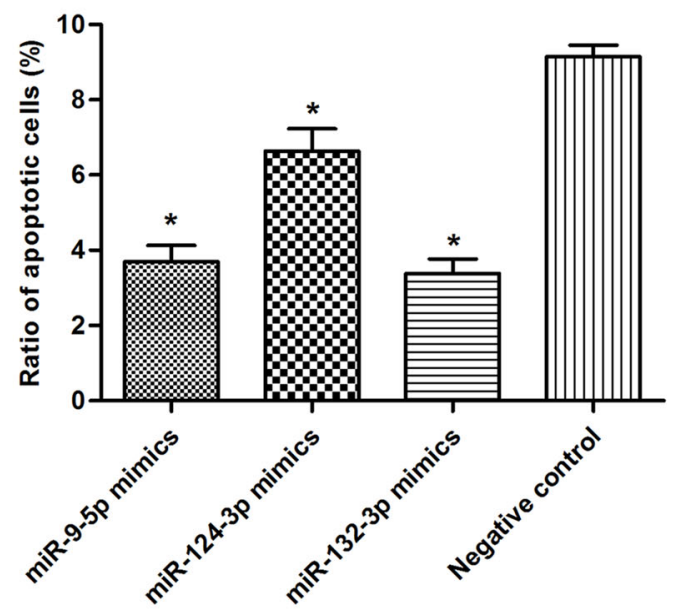

F

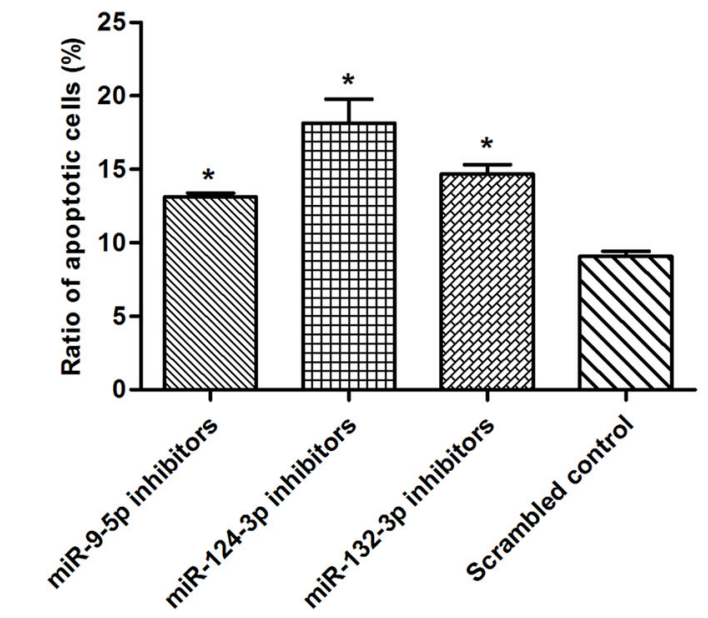

